\title{
The design of LED fish gathering lamp PC free multipoint photometer
}

\author{
Kong Xianghong ${ }^{1,3, a}$, Lu Kexiang ${ }^{1, b}$, Ji Xinlei ${ }^{1, c}$, Pan Xiang ${ }^{2, d}$, Qian Weiguo ${ }^{1,3^{*}, \mathrm{e}}$ \\ Shanghai Ocean University $\left({ }^{1}\right.$ National Engineering Research Center for Oceanic Fisheries, \\ Shanghai 201306; \\ ${ }^{2}$ Information Technology College Experimental Center of Physics, Shanghai 201306, China; \\ ${ }^{3}$ Collaborative Innovation Center for Distant-water Fisheries) \\ axhkong@shou.edu.cn blkx2011abc@163.com ${ }^{\mathrm{c}} 1039255227 @ q q . c o m{ }^{\mathrm{d}} 1528663291 @ q q . c o m{ }^{\mathrm{e}}$ wg \\ qian@shou.edu.cn
}

Keywords: Fish gathering lamp, Multipoint photometer, Zigbee

\begin{abstract}
Given the problems of testing work-load is great, data is inaccuracy and etc in the process of fish gathering lamp light illuminance testing, this article designs and proposes a efficient and fas $\mathrm{t}$ testing way to fish gathering lamp light illuminance-LED fish gathering lamp wireless synchrono us multipoint metering system, and we do the actual test in Ningtai 61 squid fishing boat. It also cac ulates and simulates the illuminance distribution of fish gathering lamp as linear light source. Ву со mparing and analyzing the actual data with theory caculation data under $10 \%$ errors, it shows that th is design not only reduce the cost of point by point measurment light illuminance and make the testi ng processes more convenient and fast; also all measuring data could be accurate and effcient.
\end{abstract}

\section{Introduction}

Light is a necessary condition for all growth and development, and it has indispensible influence for everything in this world ${ }^{\text {[1】 }}$. By making use of fish phototaxis effect ${ }^{\text {[2】 }}$, light fishing could successfully trap fish, and light gathering fish is the device of luring and collecting fish in the light fishing ${ }^{\mathbf{1 3 1}}$. The distribution of surface illumination of fish gathering lamp is always a very important

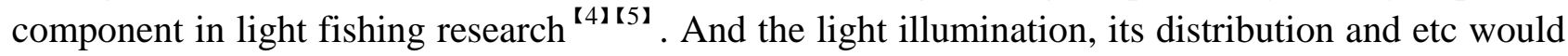
greatly affect lure and collect fish ${ }^{\mathbf{6 1 \mathbf { 1 } 7 \mathbf { l }}}$. So it appears that understanding and grasping fish gathering lamp is much more important ${ }^{\mathbf{r} 8 \mathbf{1}}$. Light detector is a kind of illumination meter based on light sensor, and it is one of the most popular light sensor. In the traditional scheme of measuring fish gathering lamp illumination distribution, we moor the light fishing boat and pre-select measuring point in the dock. After that, we keep records of illumination values at each measuring point. Because of the quite large surface wave and constantly shaking boat, especially the change of tides fluctuating and variation in water is great, the measuring data error is very big. The same data is not in the same time and space coverage, so we can not analyze information quantitatively. This article designs and manufactures a efficient, fast test method-LED fish gathering lamp PC free multipoint metering system. This system use Zigbee module that integrate CC2530 chip as the PC free transmission controller of LED fish gathering lamp photometric system.

\section{System Design}

We use the dock as the simulation sea surface to test, and in the dock plane, arrange some measuring point in the form of grid. Each measuring point is arranged to $X^{*} Y$ array mode and all has a GY-30 light sensor. The light sensor data of each line is arranged and sent by Zigbee module. That is to say, the measuring points of each line are controlled by CC2530 module. When each measuring point is measured simultaneously, the data of each line should be arranged by Zigbee module in this line and addressing the sequence. As shown in Fig.1. 


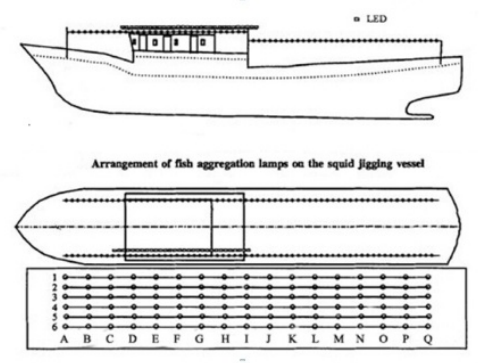

Fig.1 Measuring schematic diagram

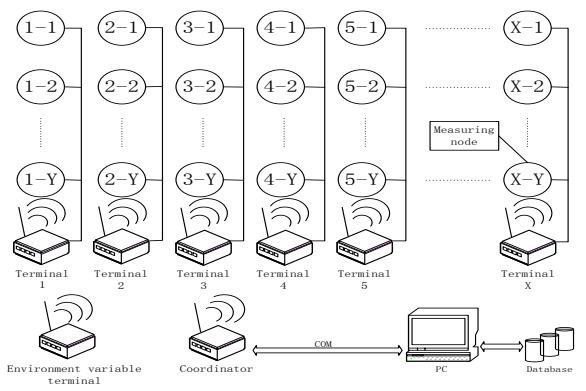

Fig.2 Synchronous transmission wireless measuring system schematic

In testing, turn on the LED fish gathering lamp, when the photon impacts to a node at GY-30 light sensor array, it would let silicon photocell generate electric current. And electric current is proportional to illumination. Through 16 bytes $\mathrm{AD}$ module transferred by amplifying circuit, electric current is transformed to digital signal and transfers the data collected by BH1750 to CC2530 singlechip through I2C communication interface. After singlechip arrange the received signal, this signal could be transferred to readable light illumination signal and encode this illumination signal based on address information of this node. The collecting, arranging and encoding of all measuring node data at CC2530 singlechip could be accomplished instantaneously. The terminal of each line transmits this line's encoded light illumination data to the coordinator in the network through Zigbee module. And coordinator transmits this data to the PC end. The data is presented on upper PC interface in the form of coordinate. All the measuring light illumination data should be stored in the database as the sequence of time, address and so on, that we could call and analyze at any moment. System scheme is showed as fig.2.

In order to ensure the reliability of the data, we must clarify the influence of environmental factors. So We add temperature and humidity sensor to monitor variable environmental factors, and it's not described in this article.

\section{System workflow}

The Communication use standard I2C protocol between the master and the BH1750. First, the sensor need to be initialized after power ups. The CC2530 transmit a group of start time series to the sensors, and the procedure is the SDA and SCL are set to high, and the SDA set up low after the delay of approximately 5 microseconds, then the SCL also set up low after the delay of approximately 5 microseconds. Then, the CC2530 transmit power-on command (function code 0x01) to the sensor. Final, the CC2530 transmit a group of stop time series to the sensors. The procedure is the SDA and SCL are separately set up low and high, and the SDA set up low after the delay of approximately 5 microseconds, and then delay for approximately 5 microseconds. Thus, the sensor initialization has completed, and waiting for sense command. When we need to sense, CC2530 transmit a group of start time series to the sensors again. And when the sensors sense the response signal, we can transmit sense command (function code of this system is 0x10). Depending on the different of measurement resolution, we need to delay for some time. Until the end of measurement, the CC2530 singlechip can receive the measurement data. The measurement data are 16 bit, and began return the high 8 bit, and then the low 8 bit. The measurement data were 
converted into a decimal number, and then divided by 1.2. This result is the final light illumination values.

The CC2530 provide a wireless transceiver based on IEEE 802.15.4 protocol, that is the RF kernel control analog radio module. The RF kernel control analog radio module achieves transmitting and receiving data by register TXFIFO and FXFIFO. When transmitting data, we write data to the TXFIFO firstly and the radio module automatically adding PHY and FCS to send data by STXON and STXONCCA. When the data has been received, the RXPKTDONE interrupt, then read RXFIFO in the interrupt service routines.

During the testing process, the collected data is sent to the terminal of signal sample module of each column (CC2530 data processing module). The terminal receives and processes the data, and transmit it to the coordinator in RF wirelessly way. Finally, the coordinator transmits data to the PC via USB. As shown in Fig.3.

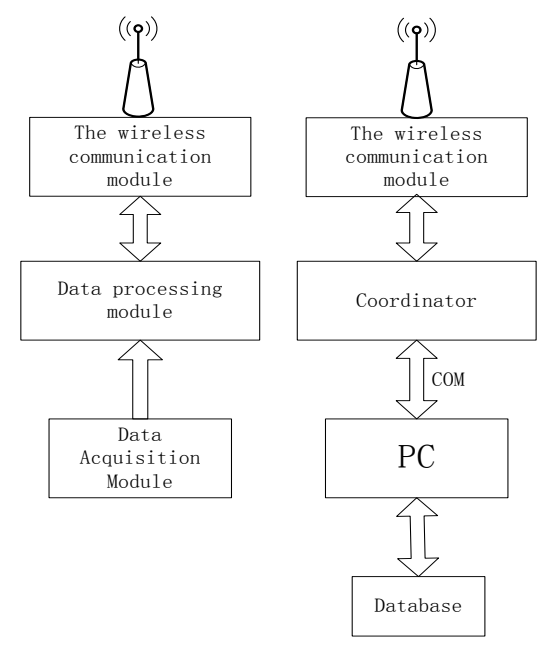

Fig.3 The work flow diagram of system

\section{The calculation and simulation of Squid jigging vessel's fishing gathering lamp illumination}

When luring the fish, LED fishing gathering lamp source is a limited strip of visible light. So LED fishing gathering lamp source can be seen as a long strip of light.

It is assumed that there is a long strip of light source, the width is $2 \mathrm{a}$, the length is $2 \mathrm{~b} . \mathrm{P}$ is the point that arbitrary point of facing light source, and the vertical distance is $r$.

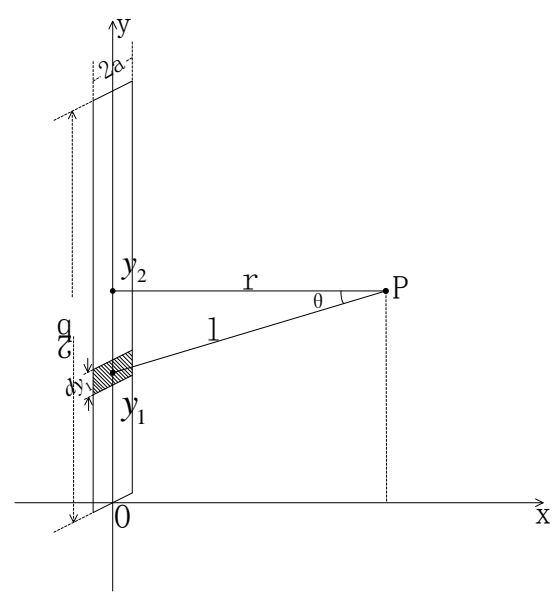

Fig.4 The calculation of squid jigging vessel's fishing gathering lamp illumination

We set light surface $S$ is cosine emitting surface and light brightness is L. The Vertical luminous intensity is $I_{0}$ for any light surface S. As shown in Fig.4, $d S=2 a d y_{1}$.

Illumination calculation of the point $\mathrm{P}$ as follows:

$I_{0}=L S$ 
According to Lambert cosine law, we get

$I_{\theta}=I_{0} \cos \theta$

By (1), (2), we have

$d I_{\theta}=2 a d y_{1} L \cos \theta$

The relationship between light illumination and intensity is

$$
E=\frac{\mathrm{I}_{\theta}}{l^{2}} \cos \theta
$$

By (3), (4), we have

$d E=\frac{d I_{\theta}}{l^{2}} \cos \theta=\frac{2 \operatorname{ady}_{1} \mathrm{~L}}{l^{2}} \cos ^{2} \theta$

It can be obtained from Fig.3 that

$$
\cos \theta=\frac{r}{l}=\frac{r}{\sqrt{\left(y_{1}-y_{2}\right)^{2}+r^{2}}}
$$

By (5), (6), we have

$$
d E=\frac{2 \mathrm{ady}_{1} \mathrm{Lr}^{2}}{\left(\left(y_{1}-y_{2}\right)^{2}+r^{2}\right)^{2}}
$$

Integrator $\mathrm{dE}$, the elongated light source for illumination $\mathrm{P}$ is

$$
E=\int_{0}^{2 b} 2 a L \frac{r^{2}}{\left(r^{2}+\left(y_{1}-y_{2}\right)^{2}\right)^{2}} d y_{1}
$$

The height of the Squid fishing boat is $4 \mathrm{~m}$, and the length of light is $50 \mathrm{~m}$. We use MATLAB software to simulate ${ }^{\text {r91 } \mathbf{1 0 1}}$, the result is shown as Fig.5.

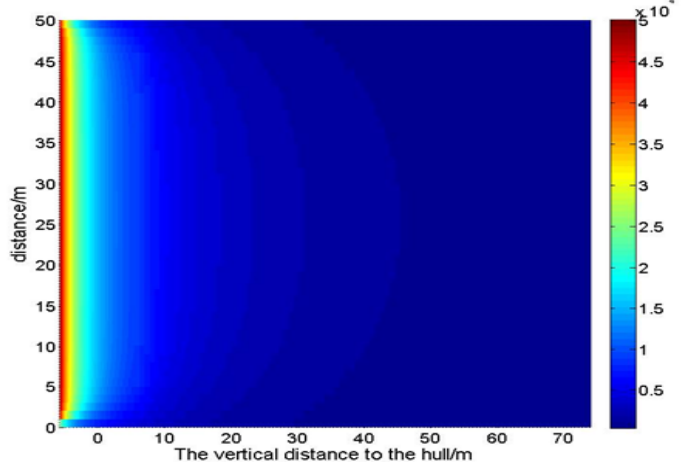

Fig.5 MATLAB simulation figure

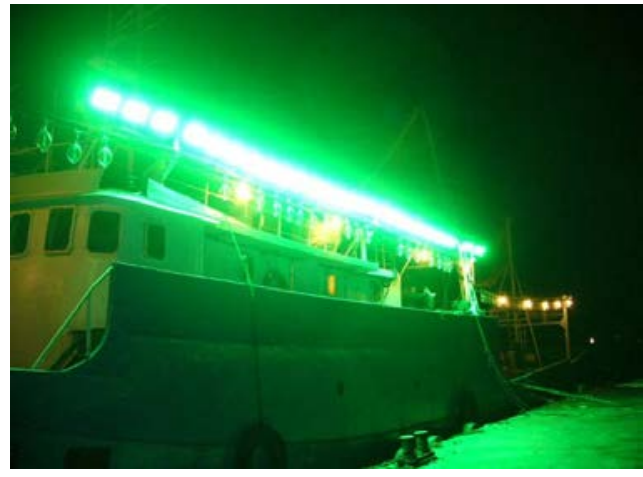

Fig.6 Measuring scenarios

\section{Conclusion}

In June, 2013, at Zhou Shan, Zhejiang fishing vessels dock, we use LED fish gathering lamp transforming light measuring system to measure Ningtai 61 squid fishing boat LED fish gathering lamp’s light illuminance distribution. As shown in Fig.6. 
By comparing the actual data with theory calculation data, we find that the overall data errors under $10 \%$. So, the result shows that the design organically mixes modern photoelectric technology, electronic micro control technology, Wireless remote signal communication technology and Visual interface data processing technology together into traditional fish gathering lamp of light fishing research. It not only reduce the cost of point by point light illuminance measurement, but also make the test procedure more convenience and effcient; and the test data would be more accurate, more favorable to reduce the error in data analysis.

\section{Acknowledgements}

This work was supported by research grants: 1. National High Technology Research and Development Plan (SS2012AA092303); 2. Shanghai science and technology commission project(2013ZY-87); 3. National engineering research center for distant-water fishery open fund projects (NERCOF2014KF03); 4. Shanghai college students' innovative projects (S20133101522).

\section{References}

[1] Okamoto T, Takahashi K, OhsawaH,et al. Application of LEDs to fishing lights for Pacific sauryp[J]. Journal of Light and Visual Environment. 2008;32(2): 88-92.

[2] Choi JS, Choi SK, Kim SJ, et al. Photoreaction analysis of squids for the development of a LED-fishing lamp[C]. In: Proceedings of the 2nd international conference on maritime and naval science and engineering. Brasov, Romania, September 24-26; 2009: 92-5.

[3] Qian W G.Study on the optimal allocation of fish aggregation lamps in squid jigging fisheries[D].Shanghai:Shanghai fisheries university,2005:1-146.

[4] Qian W G,Chen X J,Qian X L,et al.The optical characteristics of LED fish aggregation lamp(300W) and its energy efficiency[J].Marine fisheries, 2011, 33 (1): 99-105.

[5] Qian W G,WangF.Comparative study on the calculated methods of illuminate of sea surface about aggregating fish lamps[J]. Journal of Zhejiang Ocean University（Natural Science）, 2004, 23(4): 285-290.

[6] Sha F,Qian W G, Wu Z Q.The theoretical calculations of underwater irradiance of upper water fish aggregation lamps and its optimal allocation in light purse seine vessels for chub mackere(Scomberjaponicus)[J].Journal of marine sciences,2013,31 (1): 85-90.

[7] Chui X Z, Arakawa F,ArimotoG, et al.Line source modelusing in small squid fishing boats fish lamp andits illumination in the water distribution.Japanese Journal of Ichthyology.2003,69 (1): 44-51.

[8] Pan Ou. Advanced Optical Simulation(MATLAB version)-optical waveguide,laser[M].China: Beijing University Aeronautics and Astronautics Press., 2011:9-19.

[9] Qunyi Zhou, ZhaoyangHou, Ransu Liu. MATLAB visualization of university of physics[M]. China: Tsinghua University Press., 2010:226-251. 